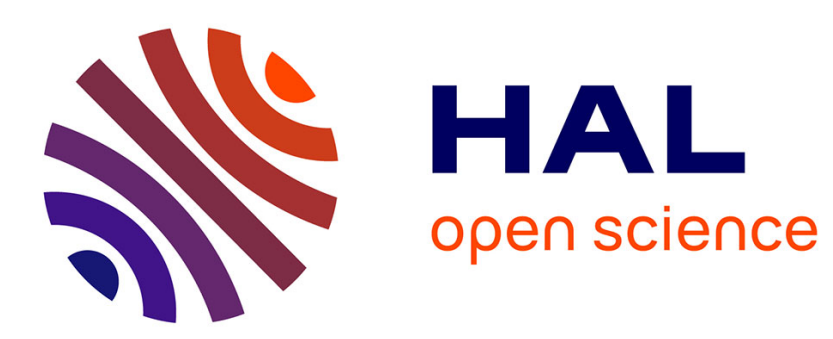

\title{
Women Writing in India: pour une histoire littéraire des femmes
}

Anne Castaing

\section{To cite this version:}

Anne Castaing. Women Writing in India: pour une histoire littéraire des femmes . Revue de Litterature Comparee, 2016, Problèmes d'histoire littéraire indienne 4, pp. 473-484. hal-01402924

\section{HAL Id: hal-01402924 \\ https://hal.science/hal-01402924}

Submitted on 6 Dec 2016

HAL is a multi-disciplinary open access archive for the deposit and dissemination of scientific research documents, whether they are published or not. The documents may come from teaching and research institutions in France or abroad, or from public or private research centers.
L'archive ouverte pluridisciplinaire HAL, est destinée au dépôt et à la diffusion de documents scientifiques de niveau recherche, publiés ou non, émanant des établissements d'enseignement et de recherche français ou étrangers, des laboratoires publics ou privés. 


\section{"Women Writing in India : pour une histoire littéraire des femmes ", in Laetitia Zecchini et Claire Joubert (dir.), Problèmes d'histoire littéraire indienne, Revue de Littérature Comparée LXXXIX, n ${ }^{\circ}$, octobre-décembre 2015 (pp. 473-484).}

\section{Anne Castaing CNRS/THALIM}

C'est au terme d'années de travaux et de prospections diverses que les critiques et militantes féministes Susie Tharu et K. Lalita publient en 1991 une anthologie magistrale, Women Writing in India ${ }^{1}$, qu'elle dédient « à toutes les auteures [qu'elles n'ont] pu inclure, et à toutes celles [qu'elles] ne connaiss[ent] pas encore $»^{2}$ : ce projet monumental, visant à dévoiler les voix et les talents littéraires féminins de l'Inde, de leurs premières traces (évaluées à 600 avant J.C. selon les auteures) à la période contemporaine, interroge à de nombreux égards les fondements même du "genre » (histoire littéraire ou anthologie ?), comme il interroge ceux, plus complexes encore, de littérature indienne et d'écriture féminine.

De ces deux volumes massifs composés d'une préface et d'une longue introduction, d'articles d'histoire littéraire («Literature of the Ancient and Medieval Period: Reading against the Orientalist Grain »; "Literature of the Reform and Nationalist Movements »; « Twentieth Century: Women Writing the Nation»), d'entrées conséquentes classées par auteures et de traductions inédites de textes littéraires, émerge de fait un sentiment de foisonnement : foisonnement des auteures (140, sélectionnées, écrivent Tharu et Lalita, à partir d'une liste initiale de 600), qui témoigne de l'ambition explicite de Tharu et Lalita de révéler des œuvres et une histoire littéraire méconnues, parfois découvertes au terme de quêtes épiques et effrénées; foisonnement des langues (onze au total, dont l'anglais), qui témoigne de l'amplitude de la zone géographique couverte; foisonnement enfin des contextes, des époques et des situations, étrangement découpés en trois "périodes » clés de l'histoire littéraire de l'Inde (Périodes ancienne et médiévale; Mouvements réformateurs et nationalistes $; \mathrm{XX}^{\mathrm{e}}$ siècle). Cette caractéristique fait certes là état d'un rapport quelque peu problématique à la périodisation, figurant à la fois des déséquilibres et des ruptures qui peuvent obturer la continuité de certaines traditions. Mais elle témoigne surtout d'une valorisation extrême de l'objet de cette anthologie: l'écriture féminine, dont les caractéristiques (marginalité, expression de la révolte, incarnation de la douleur comme condition du féminin) se soumettent peu, dans le discours de Tharu et Lalita, au contexte au sein duquel elles sont formulées, et semblent échapper aux mutations historiques et sociales qui président pourtant à l'élaboration des canons, aux sources, selon les auteures, de la marginalisation des écrits de femmes dans l'histoire littéraire.

Aussi discutable qu'il puisse être, ce constat interroge néanmoins la complexité de la relation entre histoire littéraire et histoire des femmes, alors que l'histoire culturelle et l'histoire sociale se nourrissent et se forgent mutuellement. Si le sentiment de révolte gouverne nombre d'entrées de l'anthologie, Tharu et Lalita ne manquent pas de signaler le rôle politique majeur que certaines auteures ont pu jouer, au cour du mouvement nationaliste par exemple ${ }^{3}$. Loin d'être minimisée dans ces deux volumes, la «capacité d'agir»

\footnotetext{
${ }^{1}$ Susie Tharu and K. Lalita (eds.), Women Writing in India, vol. 1 et 2, New Delhi, Oxford University Press, 1991.

${ }^{2}$ En incipit : «For all the writers we have not been able to include, for the many we do not yet know about»: Susie Tharu and K. Lalita (eds.), Women Writing in India ..., p. v.

${ }^{3}$ Il s'agit par exemple de réhabiliter la portée politique des textes et des actions de la poétesse hindi Mahadevi Verma (1907-1987), dont le rôle dans la lutte nationaliste est peu valorisé dans les histoires littéraires, et dont la révolte est réduite à des conflits existentiels que justifient l'appellation de « Mira Bai moderne ».
} 
( «agency») des écrivaines devient un motif saillant des littératures féminines, dont la volonté politique (du moins militante) est mise en exergue d'une façon parfois très prononcée. Dans cette anthologie, la question épineuse reste néanmoins celle de la capacité de Tharu et Lalita à équilibrer leur désir de combler les béances des histoires littéraires de l'Inde avec celui d'introduire les littératures indiennes dans le champ conceptuel et universel des Women's Studies, et plus particulièrement des « écritures féminines ». La question reste donc celle de la contradiction entre histoire locale et histoire globale, et de la contradiction extrêmement problématique entre féminisme occidental globalisé et littérature féminine indienne.

Au regard des tensions à l'œuvre dans cette anthologie complexe, cet article a pour ambition de souligner les questions épineuses qui président à la réalisation d'une histoire littéraire inféodée à des enjeux idéologiques et politiques, en d'autres termes d'une histoire littéraire militante. Il abordera la question des visées transnationales du féminisme occidental pour montrer la façon dont Women Writing in India, s'enracinant idéologiquement dans des terrains en apparence contradictoires, élabore un ethos subalterne qui fait écho aux positions originales de nombre d'œuvres et d'auteures dans cette anthologie et témoigne des formes d'oppression et de marginalisation complexes du sujet, qu'il soit femme, indien, littéraire, postcolonial et/ou vernaculaire.

\section{Femmes, Inde, littérature : cartographie des marges}

Aussi anodin qu'il puisse sembler, le titre de cette anthologie vise pourtant à articuler trois champs complexes : le champ du féminin ; le champ du littéraire ; le champ de l'Inde ou des études indiennes. Plus problématiques, néanmoins, les multiples champs disciplinaires qui émergent de leur combinaison : le champ de l'écriture féminine (Women + Writing); le champ de l'histoire littéraire et, plus largement, de l'histoire culturelle (Writing + India); le champ du féminisme postcolonial ou des Subaltern Studies (Women + India), selon si l'on perçoit «Women » sous l'angle du sujet genré ou celui du sujet subalterne ${ }^{4}$. Reposant sur des catégories fragiles et des domaines eux-mêmes discutables (l'écriture féminine existe-t-elle ? Qu'est-ce que la subalternité ? Qu'est-ce que l'Inde et où en sont les frontières? Où s'arrête le littéraire, dans le cadre, par exemple, des textes mystiques des nonnes du Therigatha qui ouvrent l'anthologie?), cette anthologie exige un positionnement critique clair, qui laisse penser que les questions délicates seront préalablement discutées et les choix théoriques fortement défendus.

Si la préface du recueil vise à souligner l'amplitude de l'œuvre réalisée, qui supposa un travail de recherche et d'archive considérable, un terrain difficile, la création minutieuse d'un corpus, la composition d'une équipe, enfin nombres de sacrifices au terme de choix draconiens, la longue introduction de Women Writing in India, nettement plus problématique, a pour ambition de poser les inévitables jalons critiques du recueil, notamment d'un point de vue féministe. Les premières lignes mobilisent une rhétorique qui décline de façon explicite les aspirations du projet :

What was the point, we were sometimes asked when we began work on the two volumes of Women Writing in India, of putting together an anthology such as this? Why did we think women's writing was different or that it called for special attention? Weren't women writers as much victims to social ideologies about the subordinate

\footnotetext{
${ }^{4}$ « Sujet subalterne » que, dans le langage des Subaltern Studies, Partha Chatterjee identifie aux « fragments » de la nation, c'est-à-dire les couches sociales opprimées, bâillonnées par les élites : ouvriers, petits paysans, femmes, hors-castes. Partha Chatterjee, The Nation and its Fragments. Colonial and Postcolonial Histories, Princeton, Princeton University Press, 1993.
} 
status of women as men? If we were arguing that women writers had been marginalized and their work misrepresented or misjudged, how did we suggest they should be read?

Les réponses que cette anthologie pourrait apporter, continuent Tharu et Lalita, se sont modifiées avec le temps, au fil de leurs rencontres et de leurs discussions dans le cadre de ce projet. Néanmoins, de ce paragraphe émergent certaines interrogations et termes clés, révélateurs du positionnement des auteures: la question de la spécificité de l'écriture féminine ; celle du statut subalterne des femmes ; celle, enfin, de la marginalisation (et donc, de la subalternisation) des femmes écrivains. En quelques lignes stratégiquement situées, elles y articulent en les problématisant deux des trois champs du titre : "femme» et " littérature », délaissant par le même coup le contexte, " l'Inde ».

Si Tharu et Lalita réitèrent là les vœux pieux au cœur de leur projet, conçu, écriventelles, avec le postulat que les présupposés critiques, les circonstances historiques et les idéologies ont globalement toujours été hostiles à la production littéraire féminine et ont entravé notre capacité à lire et apprécier leur travail ${ }^{5}$, on ne peut certes que reconnaître le formidable travail de réhabilitation que les auteures entreprennent, délaissant la contextualisation des œuvres et des auteurs pour s'inscrire dans les desseins plus ambitieux du vaste projet féministe transnational. Bien que le premier paragraphe de l'introduction raconte sur une dizaine de pages la disparition puis la « récupération » d'une œuvre féminine kanada du XVIII ${ }^{\mathrm{e}}$ siècle $^{6}$, la suite est motivée par une volonté explicite d'intégrer un mouvement global de réinvestissement et de réhabilitation des littératures féminines. Cette volonté se manifeste à la fois dans le choix de références littéraires puisées exclusivement dans le corpus britannique (Virginia Woolf et Mary Shelley notamment), et dans un arrière-plan critique emprunté tout autant à Julia Kristeva, Hélène Cixous ou Gayatri Spivak, qui oscille donc entre gynocritique $^{7}$, essentialisation de l'écriture féminine et volonté affirmée de déconstruire les universalismes ${ }^{8}$. L'introduction des deux volumes témoigne ainsi du désir de Tharu et Lalita de se situer au cœur d'une critique féministe complexe en tant qu'éditrices d'une histoire littéraire féministe, qu'elles extraient, en dépit de leur profession de foi maintes fois réitérée, de leur contexte culturel, social et historique.

Dans cette introduction, la mobilisation de nombre de mots-clés tels que « marginalisation », « restitution », « oppression », « parole transgressive », qu'on attribuerait plus volontiers aux Subaltern Studies qu'à la gynocritique ou au «French Feminism», suggère pourtant une volonté de contextualisation au cœur d'un projet géographiquement (l'Inde, du moins les anciennes colonies), historiquement (les années 1980-1990) et idéologiquement (marxisme et matérialisme historique) situé, qui vise à donner voix aux sans-

\footnotetext{
${ }^{5}$ Susie Tharu and K. Lalita (eds.), Women Writing in India ..., p. xvii.

${ }^{6}$ Section par ailleurs traduite ultérieurement en français et publiée séparément dans l'ouvrage collectif Enjeux contemporains du féminisme indien, Danielle Haase-Dubosc Marie E. John et al. (dir.), Paris, Éditions de la Maison des sciences de l'Homme, 2002.

${ }^{7}$ Définie dans l'ouvrage par une citation d'Elaine Showalter: «scholarship concerned with woman as the producer of textual meaning, with the history, themes, genres and structures of literature by women »: Elaine Showalter, «Towards a Feminist Poetics », Elaine Showalter (ed.), The New Feminist Criticism, London, Virago, 1986, p. 125-143, p. 128.

${ }^{8}$ Ainsi, si l'essai sur l'écriture féminine britannique The Madwoman in the Attic (1979) de Sandra Gilbert et Susan Gubar sert de modèle à l'élaboration d'une gynocritique réinventée pour le projet tant féministe, qu'historique, littéraire et social qu'est l'anthologie Women Writing in India, Tharu et Lalita mettent en garde contre les dangers d'une universalisation des œuvres littéraires, quelles qu'elles soient : « Gender subordination imaged in this domestic, middle-class terms defines the entire scope of the woman writer's world, in which, as Gilbert and Gubar image it, there appears to be only the fundamental antagonism repeatedly played out in its primal tune: that of a monolithic, unchanging patriarchy, which would seem to have no connections with other hegemonies, say, of class or race, and an equally fixed and resilient female self » (Women Writing in India..., p. 26).
} 
voix et à " laisser parler le subalterne », en l'occurrence l'écriture féminine en marge du canon littéraire masculin ${ }^{9}$. Tharu et Lalita échouent pourtant à répondre à certaines questions fondamentales que peut poser la juxtaposition, certes défendable, de la volonté d'universaliser avec celle de localiser le corpus : comment écrire l'histoire des marges sans les uniformiser ? Comment universaliser les voix et les discours (féminins, subalternes, littéraires, colonisés) sans en gommer les singularités, qu'elles soient régionales, culturelles, historiques ou linguistiques? Comment parler des femmes, des écritures et des expériences féminines sans les localiser, sans les ancrer dans un terreau qui à la fois les nourrit et les singularise, bref les marginalise ?

\section{Pour une politique de la localisation}

Le risque et la nécessité de localiser, tel est l'objet d'un brillant article de la militante féministe et poétesse Adrienne Rich, publié en 1984 :

I am to speak these words in Europe, but I have been searching for them in the United States of America. A few years ago I would have spoken of the common oppression of women, the gathering movement of women around the globe, the hidden history of women's resistance and bonding, the failure of all previous politics to recognize the universal shadow of patriarchy [...]. I would have spoken these words as a feminist who 'happened' to be a white United States citizen, conscious of my government's proven capacity for violence and arrogance of power, but a self separated from that government, quoting without second thought Virginia Woolf's statement in Three Guineas that 'as a woman I have no country. As a woman I want no country. As a woman my country is the whole world'.

This is not what I come here to say in 1984. I come here with notes but without absolute conclusions. This is not a sign of loss of faith or hope. These notes are the marks of a struggle to keep moving, a struggle for accountability ${ }^{10}$.

Texte fondamental, « Notes towards a Politics of Location » discute à la fois l'urgence et le danger de la localisation du sujet, de son discours et de sa lecture, à l'heure où le féminisme prend conscience du danger de ses visées fédératrices universalistes et cherche à se localiser, d'un point de vue tant géographique que culturel, historique, racial, social ou sexuel. La localisation («location»), selon Adrienne Rich, consiste en premier lieu à identifier sa propre position par rapport à l'objet dont elle traite, scientifiquement ou poétiquement : femme, blanche, américaine, homosexuelle, intellectuelle, terrienne, etc. Par cet effeuillage infini, que Rich déploie sur le mode de l'humour, elle met en garde contre les dangers de cette localisation quand elle aboutit à une catégorie univoque. "Qui sommesnous?», demande-t-elle en guise de conclusion; quelle catégorie peut définir cet

\footnotetext{
${ }^{9}$ Dans le premier volume de la série des Subaltern Studies, Ranajit Guha illustre bien la tentative de renversement des hégémonies entreprise par le collectif, tentative visant à donner voix aux sans-voix, et notamment aux femmes : "The historiography of Indian nationalism has for a long time been dominated by elitism - colonialist elitism and bourgeois-nationalist elitism [...] What is clearly left out of this un-historical historiography is the politics of the people. For parallel to the domain of elite politics there existed throughout the colonial period another domain of Indian politics in which the principal actors were not the dominant groups of the indigenous society or the colonial authorities but the subaltern classes and groups constituting the mass of the labouring population and the intermediate strata in town and country - that is, the people ». Ranajit Guha, «On Some Aspects of the Historiography of Colonial India », Guha Ranajit (ed.), Subaltern Studies I. Writing on South Asian History and Society. Delhi, Oxford University Press, 1982, p. 1-7.

${ }^{10}$ Adrienne Rich, «Notes Towards a Politics of Location», Lewis Reina and Mills Sara (eds.), Feminist Postcolonial Theory: A Reader [1984], New York, Routledge, 2003, p. 29.
} 
enchevêtrement de facteurs, de critères et d'événements qu'est le sujet, cette négociation interminable entre catégories multiples?

Ce danger est plus prégnant encore lorsque, comme dans le cas de Women Writing in India, il menace des catégories fragiles, marginalisées ou caricaturées : « femmes du tiersmonde »; «littérature du tiers-monde »; "femme écrivain »; " écrivain du tiers-monde ». Peut-être est-ce là ce qui justifie l'insuffisance de localisation qui caractérise l'introduction de Women Writing in India, ou peut-être s'agit-il d'une stratégie visant à faire pénétrer les marges dans le centre, à l'intérieur de ce mouvement général qu'évoque Adrienne Rich quelques années plus tôt. Enfin, peut-être Tharu et Lalita ont-elles estimé que la localisation, comme la marginalisation, ne pourraient que réduire ces voix au silence, et que la situation "Inde postcoloniale » ne permettait pas encore que se déploie l'expression émancipée du sujet, qu'il soit «femme » ou "voix littéraire », plié qu'il est sous le poids d'oppressions combinées, masculine, coloniale, nationaliste, brahmanique ?

Cette position du subalterne (ou cette «position assignée du subalterne » selon les termes de Gayatri Spivak ${ }^{11}$ ) est également ce qu'examine Adrienne Rich dans son discours sur les paradoxes délicats de la politique de la localisation. À cet égard, elle interroge l'hégémonie raciale qui caractérisa jusqu'aux années 1970 des discours féministes majoritairement blancs qui, comme le remarquait la critique et militante Ann duCille, " continuent à considérer la "blancheur" comme tellement naturelle, normative et nonproblématique que l'identité raciale est une propriété exclusivement réservée au nonblanc ${ }^{12}$. Adrienne Rich montre néanmoins comment les réponses et les contestations à cette hégémonie se virent assignées à une position marginale dans l'histoire du féminisme, où les discours blancs demeurèrent dominants car matriciels. Est dès lors gommé l'ensemble des actions d'insurrections féministes noires antérieures (dans le cadre de l'apartheid, par exemple), pourtant aux racines, selon Rich, de la naissance de mouvements majeurs comme le Black Feminism. Le danger de la localisation réside ici dans la position du sujet assignant face à l'objet assigné, de la femme blanche qui parle de (et pour) la femme noire subalterne, en gommant pour le coup les singularités de sa propre localisation, du féminin, du féminisme, et de ses modes d'expression ${ }^{13}$.

Les dangers de la localisation menacent également les tentatives d'identification des positions du texte ou du discours : à de nombreux égards, la localisation rend l'origine univoque et gomme ses amples ramifications éventuelles. Dans sa périlleuse lecture de la nouvelle de Mahasweta Devi « Stanadayini » (« La donneuse de sein », 1979), Gayatri Spivak appelle ainsi à étoffer la perspective féministe d'une perspective culturelle au sens plein du terme, à se positionner dans le texte, au cœur du vaste réseau imaginaire et idéologique dont il se nourrit. À cet égard, Spivak propose une réponse originale au tiraillement qui assaille Adrienne Rich (localiser ou ne pas localiser?), que je formulerais en des termes proprement littéraires : plutôt qu'une lecture intersectionnelle du sujet ${ }^{14}$, qui localise son interlocuteur

\footnotetext{
${ }^{11}$ « Foucault nous invite à nous rappeler que ce qui est rapporté ou dit est aussi rapporté ou dit et entraîne donc un positionnement du sujet. Il précise en outre que quiconque abordant un récit ou une histoire [...] peut et doit occuper une certaine "place du je" dans cet échange. [...] En conséquence, Foucault utilise le mot « assigné » : "la position du sujet peut-être assignée ». [...]. C'est comme si les narrativisations de l'histoire étaient structurées ou texturées comme ce que l'on appelle la littérature »: Gayatri C. Spivak, "Une représentation littéraire du subalterne : un texte d'une femme du Tiers-Monde », En d'autres mondes, en d'autres mots. Essais de politique culturelle [1987], traduit de l'anglais (États-Unis) par Françoise Bouillot, Paris, Payot 2009, p. 421466.

${ }_{12}^{12}$ Ann duCille, Skin Trade, Cambridge, Harvard University Press, 1996, p. 100.

${ }^{13}$ Voir aussi Gayatri C. Spivak, "Can the Subaltern Speak? », Laura Chrisman et Patrick Williams (eds.), Colonial Discourse and Post-Colonial Theory: A Reader [1988], New York, Columbia University Press1994, p. 66-111.

${ }^{14}$ Lecture qui viserait, selon la définition de Kimberlé Crenshaw, à l' « identification des points d'intersection du racisme et du patriarcat»: Kimberlé Williams Crenshaw, "Cartographie des marges: Intersectionnalité,
} 
dans une origine précise et immuable, Spivak privilégie une lecture intertextuelle au sens bakhtinien du terme, situant le texte au cœur d'un réseau infini de signes; réseau, écrit Mikhail Bakhtine, où « chaque mot sent la profession, le genre, le courant, le parti, l'œuvre particulière, l'homme particulier, la génération, l'âge, le jour et l'heure. Chaque mot sent le contexte et les contextes dans lesquels il a vécu sa vie sociale intense; tous les mots et toutes les formes sont habités par des intentions. Dans le mot, les harmoniques contextuelles (du genre, du courant, de l'individu) sont inévitables $\gg{ }^{15}$.

Dans En d'autres mondes, c'est tout d'abord sa propre traduction anglaise de la nouvelle de Devi que propose Spivak: "Stanadayini » raconte ainsi l'histoire de Jashoda, «mère de vingt enfants, en comptant sur ses doigts les vivants et les morts " ${ }^{16}$. "Mère de métier » («peshay janani, professional mother »), elle voue de fait ses seins à la subsistance de la marmaille des notables du village, d'une génération à l'autre, pour protéger leurs femmes des méfaits des allaitements répétés et leurs maris des attraits des servantes à la poitrine ferme. Pour produire du lait, elle doit enfanter vingt fois, et se faire nourrice de surcroît. Mais vieillissante, abandonnée par enfants et maris ingrats, Jashoda voit ses seins tarir et l'un des deux s'infecte d'une tumeur qui finit par éclater. Une plaie béante à la place du sein gauche, Jashoda s'éteindra seule dans un semi coma :

Comme le voulait la règle, le corps de Jashoda Devi, hindoue de sexe féminin, fut conservé à la morgue. À l'heure convenue, son corps fut transporté au crématorium dans une fourgonnette et elle fut brûlée, par un dom. Tout se passa exactement comme elle l'avait prévu. Jashoda est l'incarnation de Dieu: les autres font et ont fait exactement ce qu'elle pense. La mort de Jashoda est la mort de Dieu. En ce bas monde, ceux qui cherchent à égaler Dieu sont voués à une mort solitaire, abandonnés de tous (p. 196).

Or, dans En d'Autres mondes, Spivak prolonge sa traduction par une tentative d'épuisement critique de cette nouvelle complexe : dans «Une représentation littéraire du subalterne », elle effeuille les différents types de lectures auxquels "Stanadayini » pourrait inviter en tant que «texte d'une femme du tiers-monde », de "représentation littéraire du subalterne », écrit en Inde, en bengali, en 1979. Situant cette nouvelle à la confluence des " similarités et des différences", elle invite ainsi à en identifier les différentes positions, toutes singulières, et à percevoir les limites d'une lecture univoque d'un texte aussi complexe : les limites de la pensée du «féminisme marxiste», qui universalise et donc homogénéise l'exploitation des femmes; les limites du féminisme postcolonial, qui « subalternise » les productions du «tiers-monde»; les limites de l'approche de l'auteure elle-même qui, assignant à la nouvelle une position de "parabole de l'Inde après la colonisation » et soulignant la métaphorisation du corps féminin dans le projet national, gomme les stratégies de résistance des subalternes ; les limites enfin de ce que Spivak désigne comme « la grande théorie du corps féminin », incarnée par Julia Kristeva ou Luce Irigaray, qui délaissent elles aussi les spécificités de la violence faite aux corps féminins et les spécificités dans l'expression de cette violence.

politiques de l'identité et violences contre les femmes de couleur », Cahiers du genre, 2-39, [1991] 2005, p. 5182.

${ }^{15}$ Mikhail Bakhtine, « La structure de l'énoncé » [1930], dans Tzvetan Todorov, Mikhaïl Bakhtine. Le Principe dialogique, Paris, Seuil, 1981, p. 89.

${ }^{16}$ Tous les extraits en français de cette nouvelle sont issus de Mahasweta Devi, Indiennes. Rudali et autres nouvelles, traduit du bengali par Marielle Morin, Arles, Actes Sud, 2004. 
Si la lecture de Spivak révèle la complexité de la nouvelle de Devi, elle en souligne ainsi la forte localisation dans sa capacité à accueillir différents discours, tant compatibles qu'incompatibles et tant historiques que sociaux, culturels ou politiques. L'exploitation du corps féminin, au cœur de la nouvelle bien qu'elle n'en soit qu'un des multiples discours (ou l'une des multiples lectures possibles), se raconte dans un contexte où elle s'agrège à l'exploitation du Sud par le Nord, du colonisé par le colon, du tiers-monde par l'Occident, et aux réponses non moins violentes et non moins complexes à cette exploitation - la cristallisation des identités nationales, religieuses et genrées. Mais elle est aussi traversée par la multitude de discours qui s'approprient ces enchaînements de métaphores: nationalistes, orientalistes, colonialistes, féministes, subalternistes. Ainsi, conclut Spivak, le texte est habité «d'une histoire plus vaste que celle de l'auteur, du lecteur et du professeur ${ }^{17}$, ces différents sujets qui interprètent le texte et les différents discours qu'il produit.

Il est donc certain que l'insuffisance de localisation qui caractérise l'introduction de Women Writing in India condamnerait une nouvelle telle que "Stanadayini », au mieux à l'opacité, au pire au simple récit du triste destin d'une femme exploitée par les hommes puis délaissée par ses enfants, autrement dit, comme l'écrivait Spivak, au récit misérabiliste d'une subalterne du tiers-monde.

\section{Woman writing in India : provincialiser le féminisme occidental}

Le poème bengali «Bhoter Meyer Baromashi » («Le barahmasa de l'électrice», 2008) de Mallika Sen Gupta est tout aussi propice au type de lecture complexe que propose Spivak : brandissant tout autant les étendards du local et ceux de l'universel, il localise le féminisme de façon subtile par la réappropriation du langage et de la culture subalternes.

Sociologue et poétesse, Mallika Sen Gupta (1960-2011) est l'une des grandes voix de la littérature contemporaine de langue bengalie et l'auteure d'une demi-douzaine de recueils, de deux romans et de plusieurs essais sur la question du genre ${ }^{18}$. Dans les quatorze strophes du «Barahmasa de l'électrice», elle décrit les difficultés que rencontre la femme traditionnelle au quotidien en contexte rural, qui justifient, mois après mois, son incapacité matérielle d'aller voter : la fatigue liée à la chaleur ou à la faim en période de sécheresse, le froid en hiver, les maladies des enfants à la saison des pluies, la dot à réunir à la saison des mariages, les coups du mari alcoolique, les travaux qu'elle doit accomplir pour subvenir aux besoins de toute la famille, les fêtes religieuses (Id chez les musulmans ou Durga Puja chez les hindous) dont les femmes sont les piliers, etc. «Mais je voterai, conclut-elle, pour celui qui apportera le progrès ».

C'est par le détournement d'un genre littéraire appartenant au patrimoine féminin que Sen Gupta déploie le langage subalterne. Dans ce poème, elle se réapproprie la forme poétique traditionnelle barahmasa des littératures vernaculaires d'Inde du Nord, " chanson des douze mois » qui décrit en douze paragraphes, mois par mois, les transformations de la nature comme métaphores des sentiments de la récitante, très souvent une femme souffrant de l'angoisse de la séparation (viraha) d'avec son aimé. L'élément descriptif issu du paysage naturel saisonnier est ainsi combiné à l'élément pathétique, les deux formant un tout inséparable $^{19}$ : les nuages noirs de la saison des pluies peuvent-ils ainsi rappeler l'absence de

\footnotetext{
${ }^{17}$ Gayatri C. Spivak, En d'autres mondes..., p. 466.

18 Strilinga Nirmana («La construction du féminin», Kolkata, Ananda Publishers, 1994), Purush Noi Purushtantra (« Pas l'homme, mais le patriarcat», Kolkata, Vikash Grantha Bhavan, 2002), Vivahabichchinnar Akhyan, Banglar Samaj O Sahitye («Récit d'un mariage brisé : la société et la littérature bengalies », Kolkata, Papyrus, 2007).

${ }^{19}$ Voir Charlotte Vaudeville, Barahamasa. Les Chansons des douze mois dans les littératures indo-aryennes, Pondichery, Institut Français d'Indologie, 1965.
} 
l' " amant à la peau noire » (en référence au dieu Krishna, l'amant par excellence), et les chants amoureux des oiseaux au printemps attiser la douleur de la séparation. Chez Sen Gupta, néanmoins, la thématique n'est pas tant celle la souffrance existentielle de l'amante délaissée que la souffrance physique vécue par les femmes rurales au quotidien. La précarité des conditions sociales, économiques, climatiques et sanitaires constitue de fait un frein à leur empowerment, incarné dans le poème par le vote féminin :

\section{Pourquoi veux-tu ma voix, Babu, pour qui donc voterais-je?}

Dans un barahmasa, treize étapes, et un corps saturé de douleur.

A Bankura, Purulya ou dans les régions de Gaur, de Banga, de Rarha

Les sentiments de la Phullara du Barahmasa sont toujours vivants.

2. Au mois de Vaishak [avril-mai], dis-moi, Babu, comment pourrais-je donc voter?

On meurt de soif, il n'y a pas de pompe à eau.

La boue encombre le chemin sur des kilomètres.

La cruche sur la tête, vers la mare de Sonamati,

On marche en espérant l'eau, le feu au ventre [...]

11. Dans le froid de Magh [janvier-février], n'appelle pas à voter, Babu, Le froid ne convient pas du tout à mon pauvre châle en lambeaux.

Quand donc viendra l'électricité ? Quand donc les routes seront-elles en dur?

Quand donc tous les enfants sauront-ils lire?

Si je lui dis - ne frappe plus ta femme,

Il me traitera de sorcière et m'immolera ${ }^{20}[\ldots]$

Dès le premier paragraphe, la mobilisation de la figure de Phullara, épouse de Kalketu et héroïne d'un célèbre barahmasa bengali du $\mathrm{XV}^{\mathrm{e}}-\mathrm{XVI}^{\mathrm{e}}$ siècle où elle chante ses misérables conditions de vie dans une cahute forestière ${ }^{21}$, localise donc la souffrance féminine du côté non du romantisme et des affres amoureuses, mais des conditions matérielles de vie dans un contexte rural, traditionnel, modeste, indien, postcolonial, patriarcal. Le début du texte établit ainsi une filiation explicite avec une " poétique » ou une tradition de la souffrance physique comme condition féminine, et localise précisément les racines du discours militant qu'il élabore.

S'il déploie certains stéréotypes de la «femme du tiers-monde », ce poème apporte une réponse originale: le vote comme incarnation de revendications féministes universalisantes est inadapté comme motif de mobilisation dans la situation des femmes rurales traditionnelles indiennes, où les problématiques ne sont pas différées ("belated", selon le terme de Dipesh Chakrabarty ${ }^{22}$ ) mais différentes. La langue déployée par ces femmes ne peut être celle des citadines (occidentales ou non), préoccupées par des questions globales visant à l'émancipation des femmes comme groupe homogène, mais une langue puisée dans

\footnotetext{
${ }^{20}$ Traduction d'Olivier Bougnot (non publiée).

${ }^{21}$ Voir Dusan Zbavitel, « Bengali Literature », Jan Gonda (ed.), A History of Indian Literature, vol. IX, fasc. 3, part II, Wiesbaden, Otto Harrassowitz, 1978.

${ }^{22}$ Discutant le fantasme du « retard» (social, politique, culturel) présumé des sociétés non-occidentales dans le discours de l'Occident, Dipesh Chakrabarty écrit : «On pourrait dire que [...] l'idée moderne et européenne d'histoire, est venue aux peuples non-européens au XIX ${ }^{\mathrm{e}}$ siècle, sur le mode du « pas encore » [...]. Les Indiens et les Africains n'étaient pas encore assez civilisés pour pouvoir se gouverner eux-mêmes. Il fallait que du temps historique s'écoule, il fallait qu'ils puissent se développer et se civiliser [...] afin d'être considérés comme prêts à affronter cette tâche »: Dipesh Chakrabarty, Provincialiser l'Europe: la pensée postcoloniale et la différence historique, [2000], traduit de l'anglais (Inde) par Olivier Ruchet, Paris, Amsterdam, 2009, p. 39-40.
} 
un terreau peuplé de voix et d'images appartenant à la sémiotique féminine. Cette langue est celle non d'un idiome savant mais d'un bengali populaire, habité par nombre de locutions et d'expressions propres au langage domestique qui est en contexte rural traditionnel l'espace du féminin par excellence.

La mobilisation d'un ethos populaire traditionnel, véhicule de représentations éventuellement archaïques et aliénantes du féminin (métaphore de la nation, représentation de la femme soumise, exploitation du corps féminin), témoigne à la fois des singularités des revendications féministes en contexte non-occidental et d'une volonté de réappropriation des cultures vernaculaires comme espace autonome de résistance. L'originalité du féminisme déployé chez Malika Sen Gupta, comme chez d'autres auteurs ${ }^{23}$, est tout autant de s'extraire de la simple dénonciation de la domination masculine pour explorer la subjectivité des femmes traditionnelles, que d'échapper dans cette représentation au misérabilisme, qui pourtant coïnciderait avec une lecture « délocalisée » du poème. Sa pertinence réside donc la localisation du féminin par à la fois un recentrement de la ruralité comme environnement de la majorité des femmes indiennes, et une hospitalité des mythologies, des images et des fables qui nourrissent l'imaginaire des femmes en contexte traditionnel. Si la « relocalisation » des universalismes est là l'un des motifs saillants de nombre de romancières et poétesses féministes contemporaines en Inde, elle permet de valoriser l'hétérogénéité des expériences et des contextes, de valoriser les exceptions et de mettre en lumière la pluralité des types d'oppression.

La position des auteures qui recomposent une culture subalterne comme espace d'émancipation témoigne d'une certaine volonté de provincialisation de l'Europe, du moins de cette Europe métaphorique, incarnation des paradigmes du pouvoir, dont parle Dipesh Chakrabarty $^{24}$ : en édifiant un outil de mobilisation politique efficace à partir d'éléments traditionnels, populaires, mythologiques, et donc non-rationnels; en s'adressant au groupe opprimé dans sa propre langue; en repensant les catégories d'oppression et de féminisme et en érigeant la tradition en outil d'émancipation; en créant, en conséquence, un espace où le subalterne peut non seulement parler, mais également devenir sujet de sa propre histoire par sa propre culture.

La question que pose néanmoins la « localisation» par l'exploration des marges, la valorisation des petits récits et la mobilisation de cultures subalternes autonomes est certes celle du renversement de l'universalisme mais également, on le conçoit, celle de la menace d'un repli identitaire et idéologique qui les rend inadéquates dans un monde globalisé. L'exemple des dynamiques féministes locales est tout à fait significatif puisqu'en valorisant la singularité des modes d'émancipation, c'est l'émancipation elle-même, dans sa conception certes occidentale mais arrachée au terme d'un long combat, qu'elles mettent en question. On peut dès lors comprendre l'acharnement universaliste des revendications féministes européennes des années 1970, comme l'on peut comprendre les visées universalistes de Tharu et Lalita. De cette façon, et dans les termes de Gyanendra Pandey, elles négocient une subalternité (celle qui justifie l'existence même du projet, qui vise, écrivent-elles, à revaloriser des littératures mésestimées, marginalisées ou inexistantes) comme résultant d'une relation de pouvoir et d'un système hégémonique, tout en repoussant le principe de différence

\footnotetext{
${ }^{23}$ Par exemple chez Mahasweta Devi pour le bengali, ou chez la poétesse Anamika ou la romancière Mallika Sen Gupta pour le hindi.

${ }^{24}$ Explicitant le projet au cœur de son ouvrage Provincialiser l'Europe, Dipesh Chakrabarty écrit : « L'Europe que je cherche à provincialiser ou décentrer est une figure imaginaire qui, sous forme de clichés et de raccourcis, demeure profondément ancrée dans certaines habitudes de la pensée ordinaire qui sous-tendent invariablement les tentatives faites par les sciences sociales pour soulever la question de la modernité politique en Asie du Sud » (Provincialiser l'Europe..., p. 32).
} 
comme « essence » et comme «moyen de légitimer et renforcer les relations de pouvoir ${ }^{25}$. Autrement dit, l'écriture féminine n'est pas tant différente de l'écriture masculine qu'elle lui est subordonnée du point de vue de l'histoire littéraire. En déployant une histoire des littératures subalternes accolées d'un point de vue critique au projet féministe transnational, Tharu et Lalita permettent de contourner le principe essentialisant de la " différence » pour s'enraciner dans une rhétorique de l'oppression, de la marginalisation et de la subalternité. Ainsi, écrivent-elles dans les dernières lignes de l'introduction de Women Writing in India:

What we have tried to do [...] is to create a context in which women's writing can be read, not as new monuments to existing institutions or cultures (classics are, by definition, monuments), but as documents that display what is at stake in the embattled practices of self and agency, and in the making of a habitable world, at the margins of patriarchies reconstituted by the emerging bourgeoisies of empire and nation. [...] In other words, we are interested in how the efforts of these women shaped the worlds we inherited, and what, therefore, is the history, not of authority, but of contest and engagement we can claim today ${ }^{26}$.

\footnotetext{
${ }^{25}$ «Difference becomes a mark of the subordinated and subalternized, measured as it is against the purported mainstream, the 'standard' or the "'normal' [...]. It is in the attribution of difference, then, that the logic of dominance and subordination has commonly found expression. The proclamation of difference becomes a way of legitimating and reinforcing existing relations of power », Gyanendra Pandey (ed.), Subalternity and Difference. Investigations from the North and the South, London and New York, Routledge, 2011, p. 3.

${ }^{26}$ Susie Tharu and K. Lalita (eds.), Women Writing in India..., p. 36.
} 